\title{
Nuclear medicine techniques in Merkel cell carcinoma: A case report and review of the literature
}

\author{
NIKOLAOS KRITIKOS ${ }^{1}$, DIMITRIOS PRIFTAKIS ${ }^{1}$, STAVROS STAVRINIDES $^{2}$, \\ STEFANOS KLEANTHOUS $^{1}$ and ELENI SARAFIANOU ${ }^{1}$
}

\begin{abstract}
Departments of ${ }^{1}$ Nuclear Medicine and ${ }^{2}$ Gastroenterology, St. Savvas Anticancer-Oncology Hospital, Athens 11522 , Greece
\end{abstract}
Received July 9, 2014; Accepted March 25, 2015

DOI: $10.3892 / \mathrm{ol} .2015 .3377$

\begin{abstract}
Merkel cell carcinoma (MCC) is a rare and aggressive type of neuroendocrine cancer of the skin. It predominantly affects the elderly, with a predilection for the sun-exposed skin of the head and neck. Risk factors include immune-suppressing diseases, such as human immunodeficiency virus (HIV), chronic lymphocytic leukemia and multiple myeloma, organ transplantation, and the presence of the newly-identified Merkel cell polyomavirus (MCPyV). Diagnosis is based on pathological findings, primarily the immunohistochemical determination of cytokeratin 20 positivity. By contrast, staging relies on conventional imaging methods, such as ultrasonography, computed tomography $(\mathrm{CT})$ and magnetic resonance imaging, and nuclear medicine techniques, such as sentinel lymph node scintigraphy, somatostatin receptor scintigraphy (SRS), and positron emission tomography (PET)/CT with ${ }^{18} \mathrm{~F}$-fluorodeoxyglucose (FDG) or alternative radiopharmaceuticals. The treatment of MCC is primarily surgical, with possible adjuvant radiation, while the use of chemotherapy appears to be an alternative therapeutic option that is used only in specific cases. The present study describes the case of a 43-year-old HIV-positive Caucasian man with MCC located on the posterior surface of the left thigh, which was identified by cytological and histological examination of tissue sampled by fine needle aspiration and biopsy performed under CT. SRS demonstrated a high uptake of ${ }^{111} \mathrm{In}$-diethylene triamine pentaacetic acid-octreotide at the affected site. Therefore, the lesion was surgically excised, and the patient received chemotherapy and adjuvant radiotherapy. Three months subsequent to treatment, the patient underwent a PET/CT scan with ${ }^{18} \mathrm{~F}$-FDG that demonstrated uptake in the cervical
\end{abstract}

Correspondence to: Mr. Nikolaos Kritikos, Department of Nuclear Medicine, St. Savvas Anticancer-Oncology Hospital, 171 Alexandras Avenue, Athens 11522, Greece

E-mail: nikos_md@yahoo.gr

Key words: merkel cell carcinoma, nuclear medicine, positron emission tomography-computed tomography, somatostatin receptor scintigraphy, sentinel lymph node scintigraphy lymph nodes and the area of the excised lesion. These findings indicated that the disease was in remission. The aim of the present study was to highlight the value and contribution of nuclear medicine in the diagnosis, staging and follow-up, using PET/CT, octreoscan and sentinel lymph node scintigraphy, of patients with MCC, as well as the therapeutic strategy of radiolabelled somatostatin analogue scintigraphy.

\section{Introduction}

Merkel cell carcinoma (MCC) was initially described by Toker in 1972 as trabecular carcinoma of the skin in a report of five patients with unusual skin tumors (1). In 1875, the German anatomist and histopathologist Merkel termed these cells, which are localized at the basal layer of the skin and mucosa, as 'touch cells', prior to their subsequent renaming to Merkel cells (2,3). In 1978, Tang and Toker (4) identified dense core granules, a morphological hallmark of Merkel cells, in three of the original five tumors investigated by Toker using electron microscopy. Thus, it was assumed that this type of trabecular carcinoma arises from Merkel cells. However, the exact origin of $\mathrm{MCC}$ remains a controversial topic, with the two predominant theories stating that MCC descends from stem cells of neural crest origin or from epithelial cells of the epidermis. To date, the first hypothesis appears to be the most widely accepted $(3,5)$.

MCC is a rare condition with an estimated annual incidence of 0.23 cases per 100,000 individuals throughout the Caucasian population (6). However, according to age-adapted incidence rates, the occurrence of MCC increased between 1986 and 2001, exhibiting a statistically significant annual increase of $8 \%$ (7). Possible reasons for this increase include longer life expectancy, greater sun exposure and a growing number of immunocompromised individuals (6). Furthermore, there is a higher incidence in men and the elderly (6).

The etiology of MCC is unknown. However, the pioneering identification of a novel Merkel cell polyomavirus (MCPyV), which was associated with the development of MCC by Feng et al (8), led to numerous attempts to investigate viral oncogenesis. Feng et al (8) detected MCPyV in 8 out of $10 \mathrm{MCC}$ patients. Furthermore, in a study conducted by Fukutomo et al (9), frequent detection of MCPyV DNA in human papillomavirus-1 (HIV-1)-positive patients was reported, indicating that MCPyV viremia is associated with host immunity. 
Notably, it was also reported that patients with acquired immunodeficiency syndrome (AIDS) demonstrate a 13.4-fold increase in the risk of developing MCC compared with healthy individuals (10).

Additionally, evidence regarding the origin of MCC has been provided by a number of strongly associated risk factors and frequent clinical features, presented below. MCC can be challenging to diagnose and may be overlooked in the early stages, as the development tends to be asymptomatic, without pathognomonic clinical features. Typically, MCC presents as a small, firm, red to purple painless papule or nodule that grows progressively in size. MCC is predominantly observed in the head and neck region; however, alternative locations include the upper (19\%) and the lower limbs (16\%), which are associated with the best prognosis, and the trunk (11\%), a location more often associated with distant metastasis at the time of diagnosis (11). In a study conducted by Heath et al (12), the most common clinical features in 195 patients were described using the acronym AEIOU, indicating the following: Asymptomatic, which occurs in $88 \%$ of patients; expanding rapidly, which occurs in $63 \%$ of patients; immune-suppressed, including patients with AIDS or chronic lymphocytic leukemia, or those that have undergone transplantation, comprising $7.8 \%$ of patients; $>50$ and $>65$ years old, which accounts for 90 and $75 \%$ of patients, respectively; and UV-exposed and fair-skinned, which accounts for 81 and $98 \%$ of patients, respectively.

A definitive diagnosis of MCC is determined by a histopathological examination performed subsequent to biopsy, and is predominantly based on the expression of cytokeratin 20 (CK20), a protein that is highly specific for MCC (13). In a review by Jaeger et al (14), it was demonstrated that, as well as CK20, the expression of neuron-specific enolase (NSE) and neurofilament protein are specific for MCC. Other neuroendocrine markers commonly used for MCC diagnosis are chromogranin and synaptophysin, and negative markers include thyroid transcription factor-1 (TTF-1), CK7, diagnostic markers for small cell lung cancer and leukocyte common antigen for lymphoma (3). Furthermore, the differential diagnosis between MCC and malignant melanoma is based on the presence of CK20 expression, and the absence of human melanoma black 45, NKI/C3 and S-100 expression (3). Data regarding the regional and metastatic disease is obtained using various imaging techniques, including ultrasound (US), computed tomography $(\mathrm{CT})$ and magnetic resonance imaging (MRI), as well as nuclear medicine modalities. Therefore, these techniques facilitate the staging, management and follow-up of patients with MCC $(3,7)$.

Surgery is considered to be the primary treatment strategy for patients with MCC. Specifically, the National Comprehensive Cancer Network guidelines (www.nccn. org/professionals/physician_gls/f_guidelines.asp) recommend a margin of 1-2 cm for wide local excision or, alternatively, treatment with Mohs surgery. In patients treated with wide local excision, sentinel lymph node biopsy (SLNB) is performed intraoperatively, as in the present case. By contrast, SLNB should be performed prior to undergoing Mohs surgery as the surgical technique may alter the lymphatic drainage, leading to changes in sentinel node scintigraphy (5). MCC is a radiosensitive malignancy; however, the use of radiotherapy (RT) as monotherapy is typically reserved for patients that are not

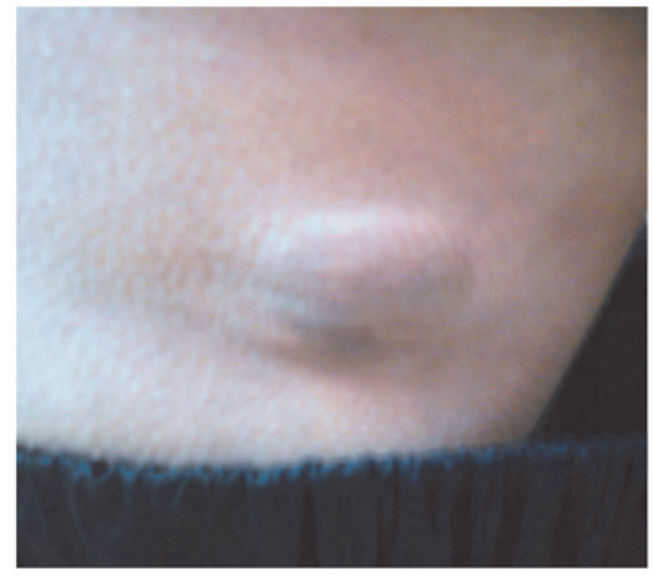

Figure 1. MCC lesion at presentation. The 4-cm lesion skin-colored lesion was located on the posterior side of the left thigh.

eligible for surgery (5). Mojica et al (15) reported a median survival time of 63 months for patients who were treated with RT as an adjuvant to surgery, compared with 45 months for those who underwent surgery alone. The treatment of MCC with chemotherapy remains under evaluation. Although MCC is considered to be a chemosensitive malignancy, a standard chemotherapeutic treatment scheme does not yet exist. Due to MCC exhibiting similar biological behavior to small cell lung cancer, chemotherapeutic regimens, such as etoposide/carboplatin or cyclophosphamide/doxorubicin/vincristine, have been used (5). Peptide receptor radionuclide therapy (PRRT) is an alternative treatment option in appropriate cases (16).

The typically poor prognosis of patients with MCC appears to be associated with the degree of expansion at the time of presentation. The aggressive course of this rare neoplasm is reflected by the following five-year survival rates: Local disease, 64\%; regional nodal involvement, 39\%; and distant metastatic disease, $18 \%$ (17). Furthermore, MCC appears to have a high three-year mortality rate of $33 \%$. This rate is $\sim 15 \%$ higher than that of the less aggressive skin cancer melanoma (12). Even following treatment, close monitoring of patients is required due to the following high recurrence rates: Local recurrence within 12 months, 30-40\%; regional recurrence within two years, $50 \%$; and distant metastasis, 36-49\% (18).

\section{Case report}

In 2012, a 43-year-old Caucasian man presented to St. Savvas Anticancer-Oncology Hospital (Athens, Greece) with a painless skin lesion on the posterior side of the left thigh that was reported to be growing in size. The patient was HIV-positive and undergoing treatment. Upon physical examination, a skin-colored lesion of 4-cm diameter was observed (Fig. 1) and MRI revealed a suspicious mitotic space-occupying lesion (Fig. 2). Thus, a cytological and histological examination with fine needle aspiration and biopsy was immediately performed under CT. The cytological profile of the lesion was consistent with MCC; however, the histological findings indicated a diagnosis of neuroendocrine carcinoma of the skin. Immunohistochemistry identified that the lesion was 
A

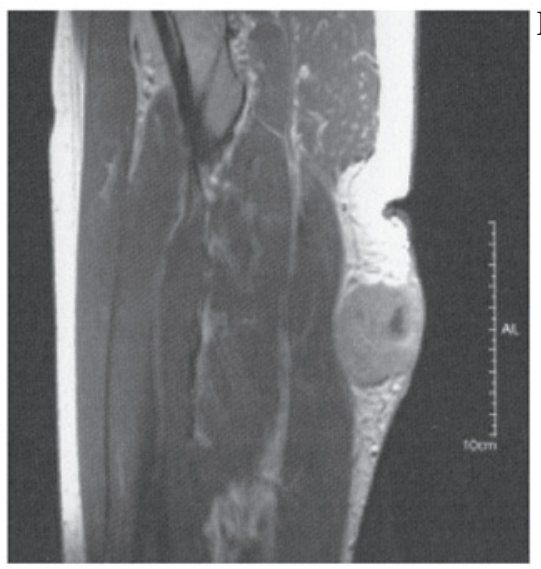

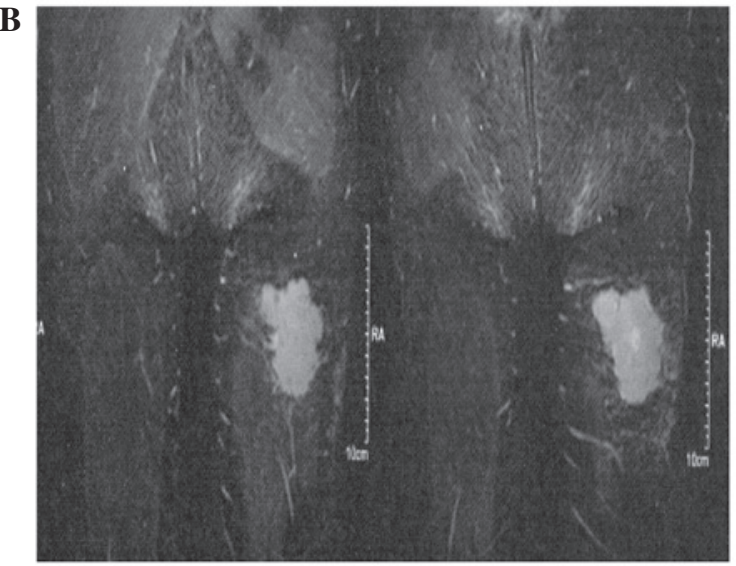

C

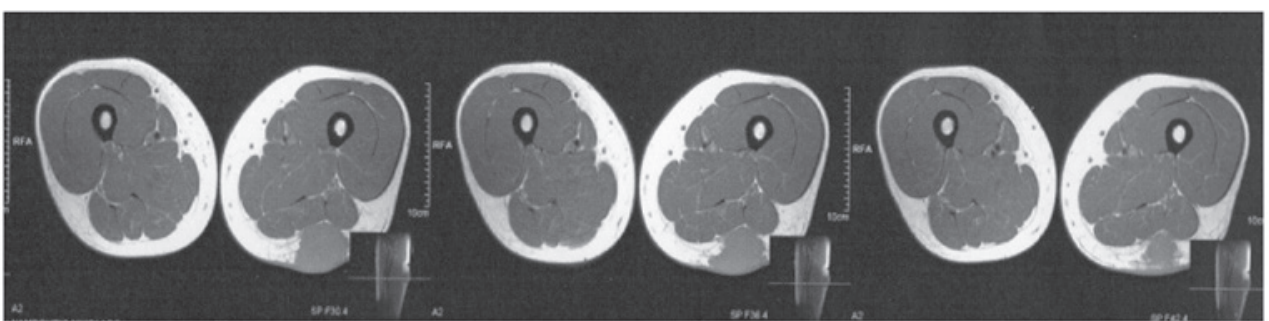

Figure 2. Magnetic resonance imaging, revealing a space-occupying lesion in the soft tissue of the posterior side of the left thigh. (A) Saggital view; (B) two coronal views posterior and anterior; and (C) three transverse views from top to bottom, of magnetic resonance imaging, revealing a space-occupying lesion in the soft tissue of the posterior side of the left thigh.

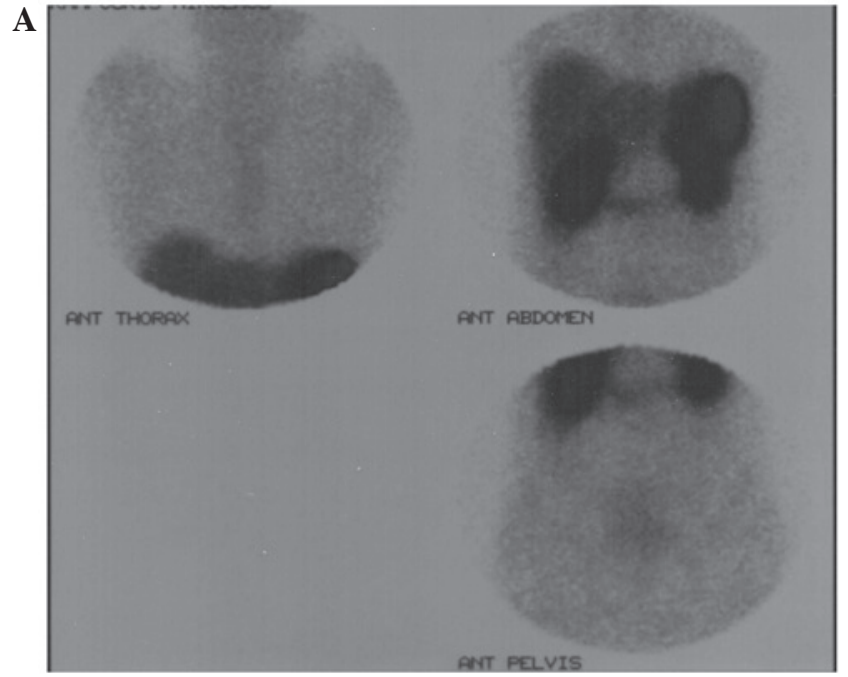

Figure 3. (A) Ant and (B) pos view of somatostatin receptor scintigraphy with ${ }^{111}$ In-diethylene triamine pentaacetic acid-octreotide, revealing overexpression

of somatostatin receptors only at the affected site. Pos, posterior; ant, anterior.
B

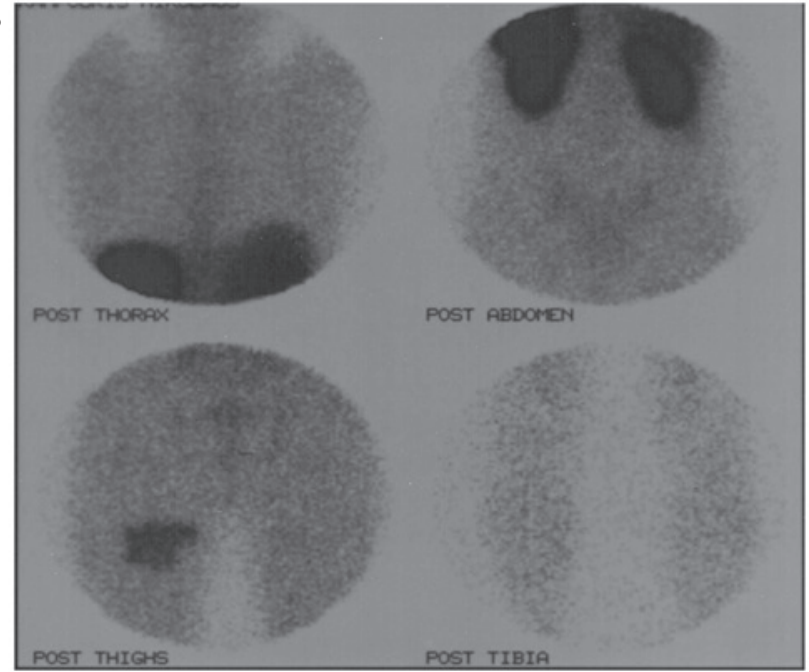

CK20(+), chromogranin(+), synaptophysin(+), NSE(+) and CK8/18(+). The patient subsequently underwent somatostatin receptor scintigraphy. High uptake of the injected radiopharmaceutical was observed in the anatomical location of the lesion, indicating overexpression of somatostatin receptor subtypes 1 and 5 (SSTR-2 and SSTR-5, respectively; Fig. 3). Considering the scintigraphy results, the patient was examined for metastatic disease using CT. No indications of distant metastasis, such as enlarged lymph nodes or lesion elsewhere on the body, were identified. Thus, wide local excision of the lesion with an intraoperative biopsy of the sentinel lymph node, identified by scintigraphy, was performed. Subsequent histological analysis determined that the node was free from malignant infiltration while the whole tumor indicated the following immunohistochemical expression pattern: $\mathrm{CK}$ AE1/AE3(+); CK20(+); synaptophysin(+); chromogranin(+); epithelial membrane antigen(+); cluster of differentiation (CD) 56(+); S100(-); CD117(-); CD99(-); CD20(-); CD2(-); CD3(-); and CD43(-). Considering the aforementioned findings, a diagnosis of tumor-node-metastasis (TNM) stage IIA (T2pNOM0) MCC was determined. The patient received 8 21-day cycles of chemotherapy with adjuvant RT. The chemotherapeutic regimen consisted of epirubicin (35 mg) on days 1-3, cyclophosphamide (400 mg) on days 1-3 


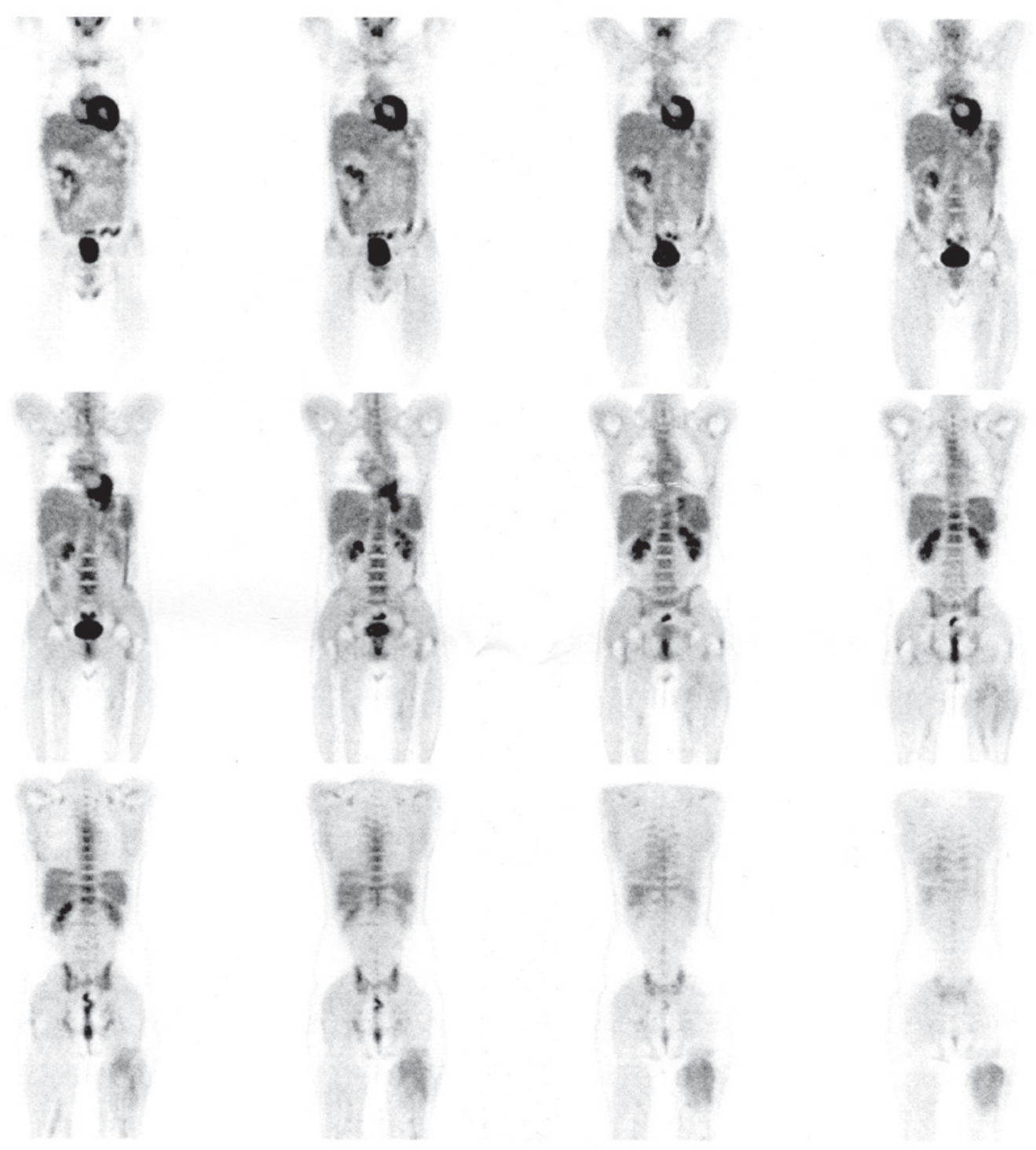

Figure 4. Whole body fluorodeoxyglucose-positron emission tomography scan performed 6 months subsequent to the completion of treatment. The scan was negative for recurrence or metastasis. The increased uptake in the cervical lymph nodes was attributed to inflammation.

and vincristine ( $2 \mathrm{mg}$ ) on day 1 , and was well-tolerated by the patient.

At follow-up, which was conducted 6 months subsequent to completion of treatment, a full body examination with ${ }^{18} \mathrm{~F}$-fluorodeoxyglucose (FDG) positron emission tomography (PET)/CT was performed. A dose of $10 \mathrm{mCi}(370 \mathrm{Mbq})$ ${ }^{18} \mathrm{~F}-\mathrm{FDG}$ was intravenously injected and imaging was performed 60 min later. Analysis of the captured images demonstrated low ${ }^{18}$ F-FDG uptake in the posterior surface of the left thigh [maximum standardized uptake value $\left.\left(\mathrm{SUV}_{\mathrm{max}}\right), 1.2\right]$, predominantly in the area of the excised lesion, which was possibly due to the recent radiation treatment (Figs. 4 and 5A). By contrast, increased ${ }^{18} \mathrm{~F}$-FDG uptake was observed in the internal jugular lymph nodes bilaterally and in lymph nodes of the left posterior cervical triangle $\left(\mathrm{SUV}_{\max }=2.6\right)$. This increase in uptake may be attributed to inflammation, acute or as a result of the patient's chronic HIV infection (Figs. 4 and 5B). According to the FDG findings, it was determined that the patient's response to treatment was complete, the possibility of recurrence was low and the disease was in remission. However, due to the aforementioned high recurrence rates, the patient continues to be monitored with regular follow-up examinations. Furthermore, in consideration of the current data, the patient is recommended to undergo ${ }^{18} \mathrm{~F}$-FDG PET/CT 12 months subsequent to treatment. Written informed consent was obtained from the patient.

\section{Discussion}

MCC is a rare and aggressive clinical entity that has exhibited increasing frequency in recent years (18). Among the various imaging methods available, nuclear medicine techniques appear to be of significant value, providing crucial information for the staging, management and follow-up of the patient (18).

Considering that regional lymph node metastases occur frequently and early in the course of the disease, sentinel lymph node scintigraphy may be used to identify lymph nodes that could accommodate micrometastases (19). The sentinel lymph node is defined as the first lymph node in a regional lymphatic basin to receive lymph flow of tumor cells from a tumor site (19). The sentinel node is the target for this 
A

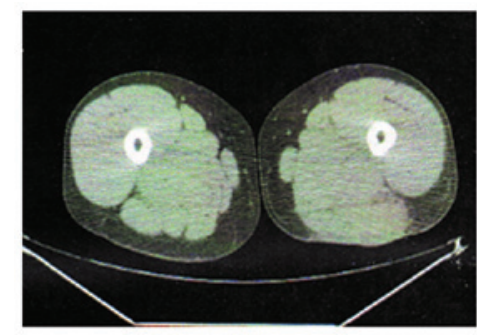

B

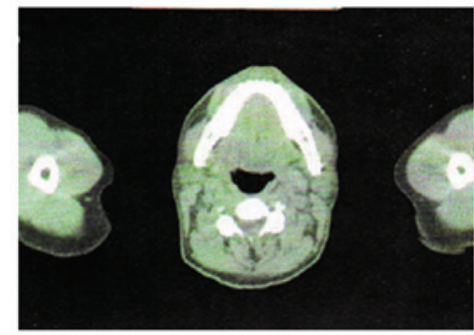

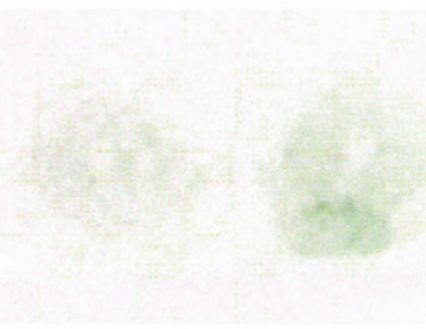
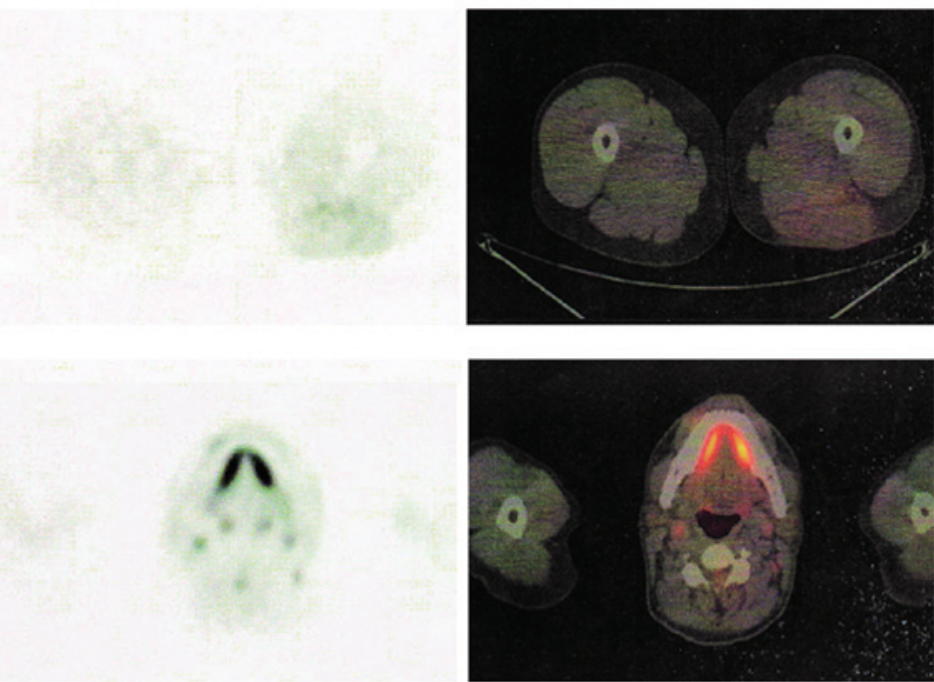

Figure 5. ${ }^{18}$ F-FDG PET-CT images. From left to right, the CT images, the PET images and the fused PET-CT images can be seen. (A) Uptake of ${ }^{18} \mathrm{~F}$-fluorodeoxyglucose (FDG) at the site of the lesion [maximum standardized uptake value $(\mathrm{SUV}$ max $)=1.2$ ], possibly due to radiation treatment. (B) ${ }^{18} \mathrm{~F}-\mathrm{FDG}$ uptake in the internal jugular lymph nodes bilaterally and in lymph nodes of the left posterior cervical triangle $\left(\mathrm{SUV}_{\max }=2.6\right)$, possibly due to inflammation.

imaging technique. The patient is subcutaneously injected with a ${ }^{99 \mathrm{~m}} \mathrm{Tc}-\mathrm{labelled}$ colloid around the lesion. Within $2 \mathrm{~h}$, the sentinel node is pre-operatively imaged and marked on the body of the patient, or intraoperatively identified with the use of a $\gamma$ probe detector, and removed for biopsy (18).

Absence of metastasis in this sentinel lymph node has a high negative predictive value, as metastases that bypass the sentinel lymph node are rare $(<2 \%)$. Therefore, if a sentinel lymph node is not infiltrated with tumor cells, it is unlikely that other nodes in the regional lymph node basin will. In the majority of cases, the pattern of lymphatic drainage is predictable when the lesion is located in the extremities. However, in the head, neck and trunk this pattern is much less predictable. In particular, unexpected nodal drainage patterns are observed in $37-84 \%$ of cases and are often missed without the use of scintigraphic guidance (20). It can take $\leq 8$ months for nodal metastases to become clinically apparent; therefore, patients with negative lymph nodes upon clinical examination alone may have occult microscopic metastatic disease (19). In 2010, the first consensus staging system for MCC was adopted by the American Joint Committee on Cancer and the International Union Against Cancer. This system was based on a study of 5823 cases conducted by Lemos et al (17) and, in contrast to previous staging systems, takes into consideration whether examination of regional lymph nodes occurred clinically or pathologically. A worse prognosis was presented for those with undetectable lymph nodes on clinical examination alone compared with those who had pathologically proven negative lymph nodes. The former, according to the new staging system, are classified as stage IB or IIB (cN0) disease, while the latter are categorized as stage IA or IIA (pN0). Considering the aforementioned studies and that regional nodal disease is a predictor of outcome, rather than crucial to the outcome, all patients with MCC should undergo sentinel lymph node scintigraphy and biopsy prior to surgery.

Nuclear medicine with PET/CT has recently gained ground in the diagnostic imaging of patients with MCC. The rationale for the application of PET in oncology is that cancer cells typically have a high metabolic activity compared with healthy tissue and use more glucose. Within tumor cells, glucose is phosphorylated by hexokinase and undergoes additional metabolism. ${ }^{18} \mathrm{~F}-\mathrm{FDG}$ is a glucose analogue that is transported into the cell where it is phosphorylated through the same mechanism as glucose, by hexokinase. However, in contrast to glucose, ${ }^{18} \mathrm{~F}-\mathrm{FDG}$ undergoes no subsequent metabolism, is unable to diffuse extracellularly and remains trapped in the cell.

Following intravenous injection, ${ }^{18} \mathrm{~F}-\mathrm{FDG}$ is rapidly distributed throughout the body and the patient undergoes imaging 40-60 min later. Clearance of the radiotracer occurs in the kidneys, and excretion through the bowel also occurs. ${ }^{18}$ F-FDG is important for the imaging of tumors with high proliferative activity. ${ }^{18} \mathrm{~F}$-FDG PET is typically more sensitive in the detection of poorly differentiated high-grade neuroendocrine tumors (NETs), with a Ki-67 index of $>20 \%$, compared with highly differentiated tumors, providing valuable prognostic information that may influence the therapeutic plan. In addition, a negative ${ }^{18} \mathrm{~F}$-FDG scan should generally be considered as a true negative result, since a negative result indicates a highly differentiated tumor and, therefore, a better prognosis. Furthermore, it has been demonstrated that the positive prognostic value of ${ }^{18} \mathrm{~F}$-FDG PET for patient outcome is better than that of traditional markers, such as Ki-67, chromogranin A and liver metastasis (21).

MCC, which presents as a rapidly growing malignancy in the majority of cases, can be imaged using ${ }^{18} \mathrm{~F}-\mathrm{FDG}$ PET/CT, allowing for differentiation between healthy and malignant tissue. Yao et al (22) reported two cases in which ${ }^{18} \mathrm{~F}-\mathrm{FDG}$ PET detected metastatic disease in subcentimeter nodes that were not detected using CT. As CT relies on tumor size and architectural change, nodes are often inaccurately characterized as benign due to a lack of enlargement. Lin et al (23) reported the increased sensitivity of ${ }^{18} \mathrm{~F}-\mathrm{FDG}$ PET for detecting MCC recurrence compared with $\mathrm{CT}$. Furthermore, in a study by Belhocine et al (24), ${ }^{18} \mathrm{~F}-\mathrm{FDG}$ PET/CT was compared with CT, MRI and bone scan alone in 11 patients. A sensitivity of $92 \%$ 
and specificity of $100 \%$ were reported, indicating that whole body ${ }^{18}$ F-FDG PET may be useful in the management of MCC patients during pretreatment staging, as well as in post-treatment follow-up. However, it was highlighted that a normal ${ }^{18} \mathrm{~F}-\mathrm{FDG}$ distribution cannot exclude the possibility of an MCC with low proliferative activity, as previously discussed. In the same study, it was reported that ${ }^{18} \mathrm{~F}-\mathrm{FDG}$ PET was able to detect a second neoplasm in 4/11 patients with MCC.

MCC is associated with secondary neoplasms, and therefore the performance of whole body ${ }^{18} \mathrm{~F}$-FDG PET may provide useful information for the restaging of patients. Concannon et al (25) conducted a retrospective review of 18 patients with $\mathrm{MCC}$ that underwent ${ }^{18} \mathrm{~F}-\mathrm{FDG} \mathrm{PET} / \mathrm{CT}$, and reported that the ${ }^{18} \mathrm{~F}-\mathrm{FDG} \mathrm{PET} / \mathrm{CT}$ findings resulted in changes in the management of nine patients. Similarly, in a study conducted by Shintani et al (26), it was reported that ${ }^{18} \mathrm{~F}-\mathrm{FDG} \mathrm{PET} / \mathrm{CT}$ findings caused two out of five patients with MCC to have their post-surgical treatment strategy altered. In a retrospective study, Peloschek et al (27) set the sensitivity of ${ }^{18} \mathrm{~F}$-FDG PET at $85.7 \%$ and the specificity at $96.2 \%$, in comparison to values of $95.5 \%$ and $89.1 \%$, respectively, that are used in conventional imaging modalities. Furthermore, in a review by Enzenhofer et al (20), ${ }^{18} \mathrm{~F}-\mathrm{FDG}$ PET/CT was recommended as first-line imaging technique for patients with MCC.

The rationale for the implementation of somatostatin receptor scintigraphy (SRS) in patients with MCC is based on the neuroendocrine characteristics of the malignancy (28). Somatostatin is a 14 amino acid peptide produced in the hypothalamus, pituitary gland, brainstem, gastrointestinal tract and pancreas. In the central nervous system, somatostatin acts as a neurotransmitter and, external to the brain, acts as a hormone that inhibits the release of growth hormone, insulin, glucagon, gastrin, serotonin and calcitonin (28). Somatostatin also acts as a tumor growth inhibitor and an angiogenesis inhibitor. Somatostatin receptors are glycoproteins of the cell membrane and are expressed in various healthy cell types, as well as in tumors of neuroendocrine origin. Five different subtypes of somatostatin receptors have been recognized at present (SSTR1-5) (28).

The radiopharmaceutical used in SRS is ${ }^{111}$ In-diethylene triamine pentaacetic acid (DTPA)-octreotide at a dose of 111-222 MBq (3-6 mCi). Octreotide predominantly binds to the SSTR2 and SSTR5 somatostatin receptor subtypes, less commonly to SSTR3, and never binds SSTR1 and SSTR4. Imaging is typically performed 24 and $48 \mathrm{~h}$ after the intravenous injection. Furthermore, clearance of the radiotracer primarily occurs through the kidneys and partially through the hepatobiliary pathway. Due to the latter clearance method, the use of laxatives and 48-h imaging is occasionally required (28).

The applications of ${ }^{111}$ In-DTPA-octreotide imaging for patients with MCC and other NETs include staging and restaging to detect primary tumors and metastatic sites, follow-up to detect relapse or progression, assessment of prognosis to predict the response to therapy, monitoring of the effects of treatment, and selection of patients to undergo PPRT (29).

An important advantage of SRS imaging compared with other conventional techniques, such as CT, MRI and US, is that this technique allows whole body imaging of the patient $(18,28)$. Considering that the majority of NETs are non-functional and appear late with tumor mass effects, distant metastasis or both, more than one modality is often required in order to gather sufficient information. In 1992, Kwekkeboom et al (30) demonstrated that SRS was associated with equivalent or greater sensitivity for imaging of MCC compared with CT. Furthermore, in a study conducted by Guitera-Rovel et al (31), 20 patients with stages I, II and III MCC exhibited SRS sensitivity of $\sim 78 \%$ and specificity of $96 \%$. In comparison with conventional modalities, SRS identified 4 out of 5 primary tumor sites, 6 out of 8 lymph node sites, no skin metastases, 2 out of 3 thoracic metastases and none of the 2 hepatic metastases. Thus, it was concluded that, in addition to conventional imaging techniques, full body SRS pre- and post-therapeutic monitoring may provide useful information for the detection of metastases and recurrence.

Limitations of SRS include non-targeted uptake in various organs, such as the liver, adrenal glands, pancreas, thyroid gland and spleen. Non-targeted uptake results in a low tumor to background ratio, making difficult to detect metastases. Additional limitations consist of the inability to detect small lesions due to suboptimal spatial resolution, relatively high cost compared with PET imaging and longer image acquisition protocol (32). In addition, SRS is limited by the diagnostic dilemma of determining whether a negative ${ }^{18} \mathrm{~F}$-FDG PET scan represents the absence of a tumor or a well-differentiated tumor that has a high possibility of expressing somatostatin receptors. Due to the aforementioned limitations of SRS, a novel imaging technique using positron-emitting somatostatin analogues, such as ${ }^{68} \mathrm{Ga}-1,4,7,10$-tetraazacyclododecane-1,4,7, 10-tetraacetic acid-( $\left.\mathrm{Tyr}_{3}\right)$-octreotate $\left({ }^{68} \mathrm{Ga}\right.$-DOTATATE), has emerged.

Following the application of SRS in the diagnosis of NETs, the next step for patients with inoperable or metastatic disease is to shrink the tumor using PPRT with ${ }^{111}$ Indium-,${ }^{90}$ Ytrium- or ${ }^{177}$ Lutetium-labeled somatostatin analogues. Proof of somatostatin receptor overexpression is required for a patient to be a candidate for such a treatment regime. Few studies have been conducted concerning this theranostic approach in patients with MCC. Schmidt et al (33) reported the cases of two patients exhibiting MCC with extensive lymph node metastases. The extent of the disease was diagnosed using ${ }^{68} \mathrm{Ga}$-DOTATATE PET/CT and after four cycles of chemotherapy, the patients underwent PRRT with ${ }^{90}$ Y-DOTATATE or ${ }^{177}$ Lu-DOTATATE in combination with capecitabine. A temporary partial response was achieved in the two patients; however, progression of the disease with fatal outcome occurred 10 and 14 months after the first symptoms occurred, respectively. For typical NETs, symptomatic control can be achieved using all the available radiolabelled somatostatin analogues. However, ${ }^{90}$ Y-DOTATOC and ${ }^{177}$ Lu-DOTATATE are the most promising, providing long-lasting responses and good survival rates (16). In addition, the use of cold somatostatin analogues is notable. In a case report by Fakiha et al (34), an 87-year-old patient diagnosed with inoperable MCC was intramuscularly injected with $15 \mathrm{mg}$ lanreotide every two weeks. Follow-up with octreoscan after 17 months revealed that the patient experienced a good quality of life, with no recurrence or side-effects.

In conclusion, MCC is a highly aggressive type of skin cancer with a high rate of metastasis and mortality. Considering 
that the median time to recurrence is nine months and that $90 \%$ of recurrences occur within the first two years of diagnosis, more frequent imaging during this period is advocated (5). Peloschek et al (27) recommended repetition of ${ }^{18} \mathrm{~F}$-FDG PET three months and one year after treatment. Considering that survival of patients with MCC is highly associated with the extent of disease at presentation, identifying patients earlier and performing adequate staging is desirable. Furthermore, nuclear medicine in combination with various evaluation methods, such as sentinel lymph node scintigraphy, SRS and PET/CT, appears to provide additional functional information regarding MCC. This additional information may have significant impact on the selection of the treatment strategy and therefore result in an optimal outcome. At present, there continues to be no imaging algorithm for MCC and the preferred modality has yet to be established; therefore, additional studies are required. Due to the rarity of the malignancy and despite good understanding of a large extent of the biology of this neoplasm, there continue to be numerous unanswered questions regarding MCC. The current study proposes that greater understanding of this disease entity can be achieved by thoroughly evaluating each individual case. The purpose of the present study was to establish the role of nuclear medicine techniques, as applied in the current case, and to contribute to an evidence-based imaging approach to MCC. Specifically, sentinel lymph node biopsy guided by the scintigraphy, along with the preoperative somatostatin receptor scintigraphy contributed to the accurate staging of the patient, and thus influenced the therapeutic decision. Additionally, ${ }^{18}$ F-FDG PET was used for the whole body post-therapeutic monitoring of metastases and recurrence.

\section{References}

1. Toker C: Trabecular carcinoma of the skin. Arch Dermatol 105 107-110, 1972.

2. Merkel F: Tastzellen und Tastkörperchen bei den Hausthieren und beim Menschen. Archiv für Mikroskopische Anatomie 11: 636-652, 1875.

3. Erovic I and Erovic BM: Merkel cell carcinoma: The past, the present and the future. J Skin Cancer 2013: 929364, 2013.

4. Tang CK and Toker C: Trabecular carcinoma of the skin: An ultrastructural study. Cancer 42: 2311-2321, 1978.

5. Han SY, North JP, Canavan T, et al: Merkel cell carcinoma. Hematol Oncol Clin North Am 26: 1351-1374, 2012.

6. Duprat JP, Landman G, Salvajoli JV and Brechtbühl ER: A review of the epidemiology and treatment of Merkel cell carcinoma. Clinics (Sao Paulo) 66: 1817-1823, 2011.

7. Becker JC: Merkel cell carcinoma. Ann Oncol 21 (Suppl 7): vii81-vii85, 2010.

8. Feng H, Shuda M, Chang Y and Moore PS: Clonal integration of a polyomavirus in human Merkel cell carcinoma. Science 319: 1096-1100, 2008

9. Fukumoto H, Sato Y, Hasegawa $\mathrm{H}$ and Katano H: Frequent detection of Merkel cell polyomavirus DNA in sera of HIV-1positive patients. Virol J 10: 84, 2013.

10. Engels EA, Frisch M, Goedert JJ, et al: Merkel cell carcinoma and HIV infection. Lancet 359: 497-498, 2002.

11. Agelli M and Clegg LX: Epidemiology of primary Merkel cell carcinoma in the United States. J Am Acad Dermatol 49: 832-841, 2003.

12. Heath M, Jaimes N, Lemos B, et al: Clinical characteristics of Merkel cell carcinoma at diagnosis in 195 patients: The AEIOU features. J Am Acad Dermatol 58: 375-381, 2008.
13. Moll R, Löwe A, Laufer J and Franke WW: Cytokeratin 20 in human carcinomas. A new histodiagnostic marker detected by monoclonal antibodies. Am J Pathol 140: 427-447, 1992.

14. Jaeger T, Ring J and Andres C: Histological, immunohistological and clinical features of merkel cell carcinoma in correlation to merkel cell polyomavirus status. J Skin Cancer 2012: 983421, 2012.

15. Mojica P, Smith D and Ellenhorn JD: Adjuvant radiation therapy is associated with improved survival in Merkel cell carcinoma of the skin. J Clin Oncol 25: 1043-1047, 2007.

16. Teunissen JJ, Kwekkeboom DJ, Valkema R and Krenning EP: Nuclear medicine techniques for the imaging and treatment of neuroendocrine tumours. Endocr Relat Cancer 18 (Suppl 1): S27-S51, 2011.

17. Lemos BD, Storer BE, Iyer JG, et al: Pathologic nodal evaluation improves prognostic accuracy in Merkel cell carcinoma: Analysis of 5823 cases as the basis of the first consensus staging system. J Am Acad Dermatol 63: 751-761, 2010.

18. Nguyen BD and McCullough AE: Imaging of Merkel cell carcinoma. Radiographics 22: 367-376, 2002.

19. Arruda EP and Higgins KM: Role of sentinel lymph node biopsy in the management of merkel cell carcinoma. J Skin Cancer 2012: 176173, 2012.

20. Enzenhofer E, Ubl P, Czerny C and Erovic BM: Imaging in patients with merkel cell carcinoma. J Skin Cancer 2013: 973123, 2013.

21. Panagiotidis $\mathrm{E}$ and Bomanji $\mathrm{J}$ : Role of ${ }^{18} \mathrm{~F}$-fluorodeoxyglucose PET in the study of neuroendocrine tumors. PET Clin 9: 43-55, 2014.

22. Yao M, Smith RB, Hoffman HT, et al: Merkel cell carcinoma: Two case reports focusing on the role of fluorodeoxyglucose positron emission tomography imaging in staging and surveillance. Am J Clin Oncol 28: 205-210, 2005.

23. Lin O, Thomas A, Singh A and Greenspan B: Complementary role of positron emission tomography in merkel cell carcinoma. South Med J 97: 1110-1112, 2004.

24. Belhocine T, Pierard GE, Frühling J, et al: Clinical added-value of ${ }^{18}$ FDG PET in neuroendocrine-merkel cell carcinoma. Oncol Rep 16: 347-352, 2006.

25. Concannon R, Larcos GS and Veness M: The impact of (18)F-FDG PET-CT scanning for staging and management of Merkel cell carcinoma: Results from Westmead Hospital, Sydney, Australia. J Am Acad Dermatol 62: 76-84, 2010

26. Shintani SA, Foote RL, Lowe VJ, et al: Utility of PET/CT imaging performed early after surgical resection in the adjuvant treatment planning for head and neck cancer. Int J Radiat Oncol Biol Phys 70: 322-329, 2008.

27. Peloschek P, Novotny C, Mueller-Mang C, et al: Diagnostic imaging in Merkel cell carcinoma: Lessons to learn from 16 cases with correlation of sonography, CT, MRI and PET. Eur J Radiol 73: 317-323, 2010.

28. Ziessman H, O'Malley JP and Thrall JH: Nuclear Medicine: The Requisites. 3rd Edition. Mosby, Maryland Heights, MO, USA, pp279-281, 2006.

29. Pepe G,Bombardieri E, Lorenzoni A and Chiti A: Single-photon emission computed tomography tracers in the diagnostics of neuroendocrine tumors. PET Clin 9: 11-26, 2014.

30. Kwekkeboom DJ, Hoff AM, Lamberts SW, et al: Somatostatin analogue scintigraphy: A simple and sensitive method for the in vivo visualization of Merkel cell tumors and their metastases. Arch Dermatol 128: 818-821, 1992.

31. Guitera-Rovel P, Lumbroso J, Gautier-Gougis MS, et al: Indium-111 octreotide scintigraphy of Merkel cell carcinomas and their metastases. Ann Oncol 12: 807-811, 2001.

32. Ambrosini V and Fanti S: 68Ga-DOTA-peptides in the diagnosis of NET. PET Clin 9: 37-42, 2014.

33. Schmidt MC, Uhrhan K, Markiefka B, et al: (68)Ga-DotaTATE PET-CT followed by Peptide Receptor Radiotherapy in combination with capecitabine in two patients with Merkel Cell Carcinoma. Int J Clin Exp Med 5: 363-366, 2012.

34. Fakiha M, Letertre P, Vuillez JP and Lebeau J: Remission of Merkel cell tumor after somatostatin analog treatment. J Cancer Res Ther 6: 382-384, 2010. 